\title{
Two Types of Micromachined Vibratory Gyroscopes
}

\author{
Andrei M. Shkel \\ Mechanical \& Aerospace Engineering \\ University of California - Irvine \\ Irvine, CA, USA \\ E-mail: ashkel@uci.edu
}

\author{
Cenk Acar \\ BEI Systron Donner Automotive Division \\ Concord, CA, USA \\ E-mail: cacar@systron.com
}

\author{
Chris Painter \\ BAE Systems Inertial Products \\ Westlake Village, CA, USA \\ E-mail: Chris.Painter@baesystems.com
}

\begin{abstract}
Micromachined Vibratory Gyroscopes (MVG) can be classified into two broad types, angle gyroscopes (or Type I) and rate gyroscopes (or Type II). Gyroscopes of the first type measure orientation angles directly, while gyroscopes of the second type measure rotational rate. Most MVG implementations to date are found exclusively in the angular rate measuring variety. This paper introduces a unified approach for description of MVGs, discusses design challenges, and introduces designs and experimental results demonstrating MVGs of both types. We demonstrate that even though challenging, Type I devices can be implemented using conventional micromachining technologies. This type of micromachined devices is new, unexplored, and may potentially enable high performance micro-scale gyros. Low-grade Type II gyroscopes have been already commercially successful. We summarize our results on Type II wide-bandwidth gyro concept and demonstrate that inherent robustness against parameter variation can be effectively achieved structurally, shifting the complexity from the control electronics to the structural design of Type II MVGs
\end{abstract}

\section{INTRODUCTION}

Almost all reported to date Micromachined Vibratory Gyroscopes (MVG) are of Type II. They operate on the Coriolis principle of a vibrating proof mass suspended above the substrate. The proof mass is supported by anchored flexures, which serve as the flexible suspension between the proof mass and the substrate, making the mass free to oscillate in two orthogonal directions: the drive direction and the sense direction. In Type II implementation, the overall dynamical system is simply a two degrees-of-freedom (2-DOF) massspring-damper system, where the drive direction is typically excited by the electrostatic drive forces to a constant amplitude of oscillation, and the sense direction is excited by the rotationinduced Coriolis force. This is the most common type of vibratory gyroscopes, but is not the only one possible on the micro-scale [10].

Type I MVG was first introduced in [1], Figure 1(b). Type I gyroscope is also of the vibratory type, but in contrast to Type II is able to measure the angle of rotation directly, eliminating the need for numerical integration of the angular rate signal when information about the object orientation is required. Type I gyroscopes are two-dimensional isotropic resonators that are designed to transfer the energy between its principal axes of elasticity. In their operation, object rotation and free oscillations of an isotropic mass-spring system induce precession of the line of oscillation, with the angle of precession equal exactly to the angle of object rotation, [1]. The angle can be determined from the instantaneous position and velocity by the relationship given in Figure 1(a).

In Type II gyroscopes, rotation and constant amplitude of oscillation of a mass-spring system along the drive axis induces response along the sense axis, Figure 3(a). The angular velocity can be determined by measuring the steady-state deflection of the mass along the sense axes, Figure 3(a). The conventional Type II gyroscopes are generally designed to operate at or near the peak of their resonance curve, which makes the system to be very sensitive to variations in system parameters. Two major design concepts explored by this group are reviewed in this paper, both allowing to achieve a dynamical system with a wide-bandwidth frequency response.

\section{DYNAMICS OF VIBRATORY GYROSCOPES}

Simplified dynamic equation of motion of a broad class of Micromachined Vibratory Gyroscopes can be described in non-inertial coordinate frame as

$$
\begin{aligned}
& m \ddot{x}=-c_{x} \dot{x}-k_{x} x+m\left(\Omega^{2} x+2 \Omega \dot{y}+\dot{\Omega} y\right)+F_{x} \\
& m \ddot{y}=-c_{x} \dot{y}-k_{y} y+m\left(\Omega^{2} y-2 \Omega \dot{x}-\dot{\Omega} x\right)+F_{y}
\end{aligned}
$$

where $\Omega$ is the angular velocity of the coordinate frame, $m$ is the mass, $c_{x}$ and $c_{y}$ are damping constants, $k_{x}$ and $k_{y}$ are stiffness constants, and $F_{x}$ and $F_{y}$ are additional externally applied forces to the mass with respect to the inertial coordinate frame.

Gyroscopes are typically designed for operation under constant rate conditions $(\dot{\Omega}=0)$ and since the natural frequencies of gyroscopes are typically in the range of $1 \times 10^{4}$ to $1 \times 10^{5}$ $\mathrm{rad} / \mathrm{sec}$ and the measured rotational rates are typically on the order of $1 \mathrm{rad} / \mathrm{sec}, \omega_{x}^{2} \gg \Omega^{2}$ and $\omega_{y}^{2} \gg \Omega^{2}$. Additionally, most gyroscopes are designed such that the two natural frequencies and damping ratios are ideally identical $\left(\omega_{x}=\omega_{y}=\omega_{n}, \zeta_{x}\right.$ $=\zeta_{y}=\zeta$ ). Based off these typical operational considerations, the governing equation of motion of MVG can be simplified to

$$
\begin{aligned}
\ddot{x}+\frac{\omega_{n}}{Q} \dot{x}+\omega_{n}^{2} x-2 \Omega \dot{y} & =\frac{F_{x}}{m} \\
\ddot{y}+\frac{\omega_{n}}{Q} \dot{y}+\omega_{n}^{2} y+2 \Omega \dot{x} & =\frac{F_{y}}{m}
\end{aligned}
$$


where $Q$ is the quality factor, which is defined as the ratio of energy stored per oscillation cycle to energy lost per oscillation cycle $(Q=1 / 2 \zeta)$. In a basic formulation, Equation (2) describes the ideal dynamics of a vibratory gyroscope.

\section{TYPE I GYROSCOPES}

A Type I gyroscope is defined as a gyroscope which measures absolute angles of rotation. The ideal operation of a Type I gyroscope requires the free oscillation of the mass. In order to fulfill this criteria, it is necessary to utilize an energy sustaining feedback control which cancels the effects of damping. Such control architectures are discussed in [3], [8], [5]. Under this assumption of a zero damping condition, the only perturbations are Coriolis force. When an isotropic 2 dimensional oscillator (Foucault pendulum, an isotropic shell with axial symmetry, or any mass-spring system with equal natural frequencies in two orthogonal directions) is displaced from their position of equilibrium, then for an observer in the rotating frame, due to the Coriolis force, the energy of vibration will be transferred between the two principal axes of elasticity. Equivalently, the line of oscillation of the resonator will precess in the noninertial coordinate frame. This phenomena is illustrated in Figure 1. It can can be shown that the orientation $\phi$ of the elliptical trajectory of the mass remains constant in inertial space by the fact that $\phi$ exactly follows the negative angle of rotation

$$
\phi=-\int \Omega d t
$$

The orientation angle $\phi$ can be directly determined from the instantaneous position and velocity by the relationship

$$
\tan 2 \phi=\frac{2\left(\omega_{n}^{2} x y+\dot{x} \dot{y}\right)}{\omega_{n}^{2}\left(x^{2}-y^{2}\right)+\left(\dot{x}^{2}-\dot{y}^{2}\right)}
$$

One of the most successful vibratory gyroscopes on the inertial navigation market is the Hemispherical Resonance Gyroscopes (HRG). This device is operating on the principle of elastic waive precession described above. The sensing element of HRG is a precision shell made out of quartz. Even though desirable, it is not feasible to implement a shell on the micro-scale with required precision for Type I operation. In [1], we proposed a non-shell implementation of Type I gyroscope, and thus made it feasible to implement the concept on the micro-scale. It was demonstrated that an isotopic 2 dimensional resonator has the same dynamics as the isotropic symmetric shell and thus can be used as Type I, or angle measuring gyroscope. There is a fundamental difference between the shell and the isotropic resonator behavior. In the case of a shell a standing waive is excited on the surface of the shell and precession of the elastic waive is detected and related to the object rotation. In the case of the resonator, however, the standing waive is replaced by vibration of the resonator itself and the transfer of energy, or precession of the vibration pattern, is used for detection of the object rotation.
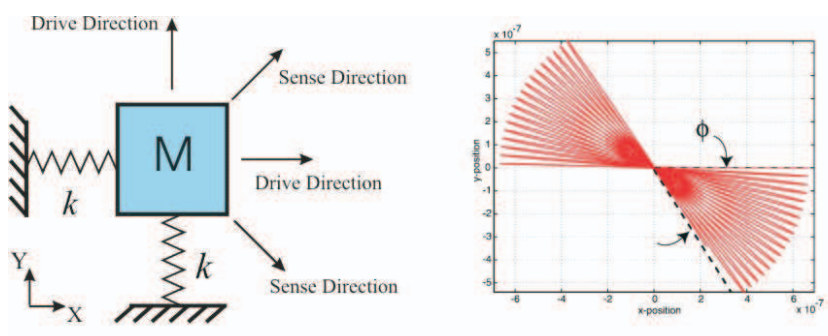

Equation of Motion

$m \ddot{x}+k x+2 m \Omega \dot{y}=0$

$m \ddot{y}+k y-2 m \Omega \dot{x}=0$

Angle of Rotation:

$\tan \phi=\frac{2\left(\omega_{n}^{2} x y+\dot{x} \dot{y}\right)}{\omega_{n}^{2}\left(x^{2}-y^{2}\right)+\left(\dot{x}^{2}-\dot{y}^{2}\right)}$

(a)

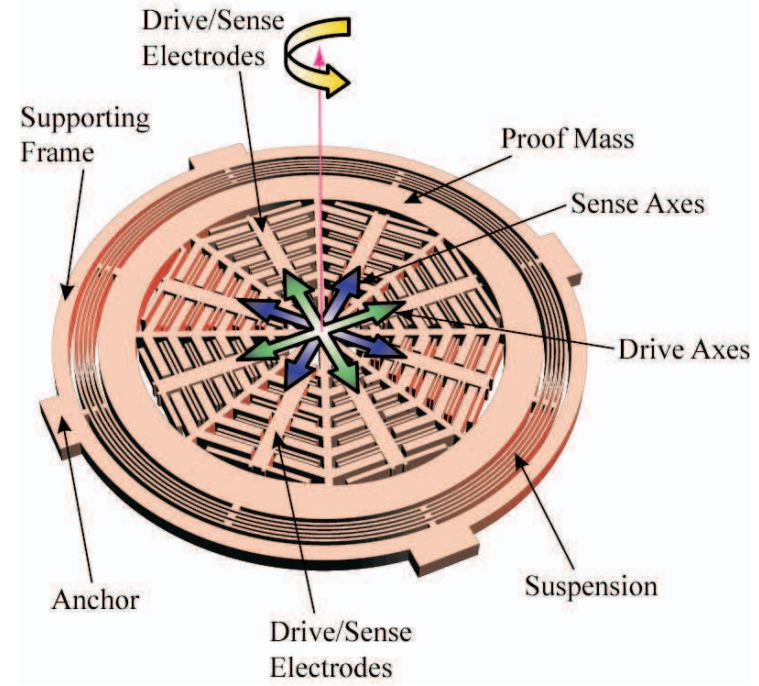

(b)

Fig. 1. (a) Ideal Response of Type I gyroscope; (b) A design of Type I gyroscope

\section{A. Design}

Type I MVGs generally consist of a mass connected to a suspension system, which is then in turn rigidly connected to a substrate below via anchors. Under ideal design, the suspension has isotropic stiffness with identical principal axes of elasticity, providing the same stiffness in all directions. The device is actuated electrostatically using fixed parallel plate electrodes anchored to the substrate below and interwoven throughout the mass.

Type I devices are fundamentally free oscillating 2 dimensional resonators. To detect the precession (or energy transfer), the detection scheme is designed to measure both position and velocity of the mass within a two dimensional operating plane. In our implementation, the Type I sensing scheme uses transimpedance configuration for velocity detection and an additional cascaded integrator for position detection. The control architecture for the z-axes gyroscope uses two orthogonal pairs of parallel plate drive electrodes. The orthogonal pairs of drive and sense electrodes are oriented with $45^{\circ}$ shift, Figure 2(a). In order to obtain the true positions and velocities, the outputs 
from the sense electrodes is transformed by a $45^{0}$ rotational transformation. The control electronics includes the feedforward and feedback controls for anisoelasticity compensation and two-dimensional damping feedback compensating control. For details on control implementation please see [5].

\section{B. Results}

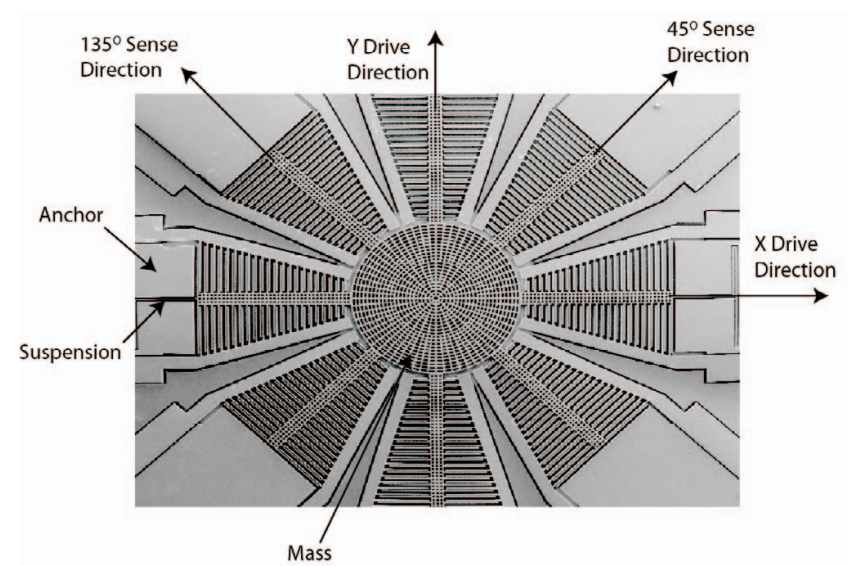

(a)

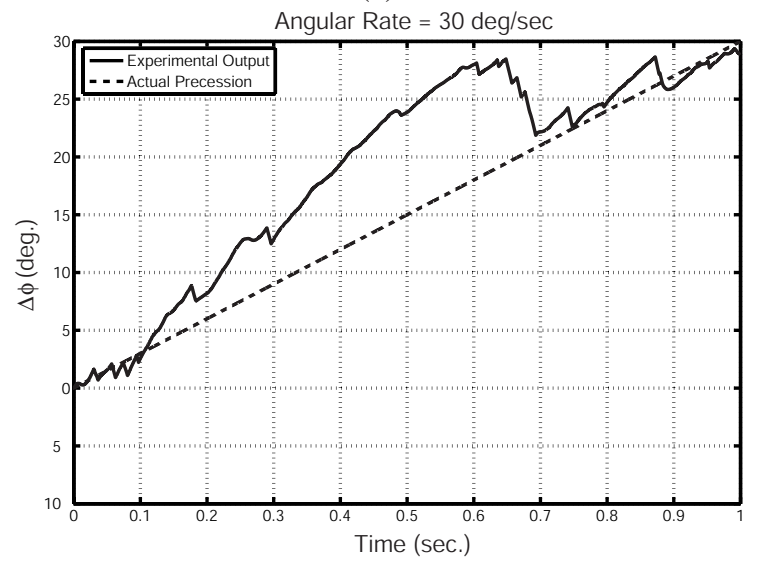

(b)

Fig. 2. (a) SEM image of a Type I gyroscope; (b) Measured precession of a Type I gyroscope

A family of Type I prototypes were implemented using SOI Bulk micromachining technology and characterized on a rate table, Figure 2(a). The devices were initially driven in an open loop mode using an $\mathrm{AC}$ voltage along $0^{\circ}$ and $45^{\circ}$ directions. In the presented experiment, the angular rate of $30^{\circ} / \mathrm{sec}$ was applied and response of the device was measured using parallel plates electrostatic detection method. In the rate table experiments, the fabricated device demonstrated rate integrating properties, responding proportionally to the angle of rotation. The observed overshooting of the precession angle is explained by step-changes in angular velocity and uncompensated frequency splits between the primary axes of elasticity. The largest contribution to the unstable nature of the precession pattern is the phase stability of the control loop.

\section{TyPE II GyROSCOPES}

\section{A. Principle}

A Type II gyroscope is defined as a gyroscope which measures rate of angular rotation. Type II gyroscopes are designed with a defined "drive" axis along which the device is continually driven using applied force $F_{x}$ and a defined "sense" axis orthogonal to the drive axis where Coriolis induced motion is sensed, Figure 3(a). In this discussion, $x$ axis is defined to be the drive axis and $y$-axis is defined to be the sense axis. Type II gyroscopes are driven in either open loop mode, where the applied force is a state independent sinusoidal input $F_{x} / m=V_{D} \sin \omega_{n} t$, or in closed loop mode, where a feedback system maintains a constant amplitude of oscillation along the drive direction, [9].

In the case of the closed loop control, assuming external force $F_{y}$ is zero along the sense direction, substitution of a constant drive amplitude $x(t)=X_{D} \sin \omega_{n} t$ into the $Y$ motional dynamics of Equation (2) gives the Coriolis force induced output steady state $y$-deflection amplitude as

$$
|y(t)|=\frac{2 X_{D} \Omega Q}{\omega_{n}}
$$

This shows that the output sense deflection is proportional to the input angular rate.

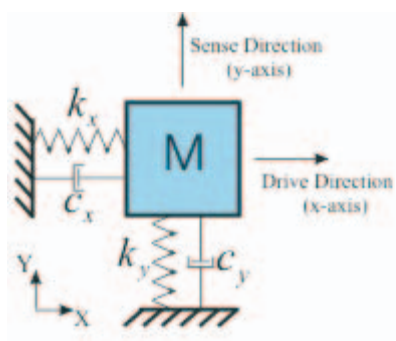

Equation of Motion

$m \ddot{x}+c_{x} \dot{x}+k_{x} x=F_{\mathrm{e}} \sin \omega t$, $m \ddot{y}+c_{v} \dot{y}-2 m \Omega \dot{x}+k_{y} y=0$

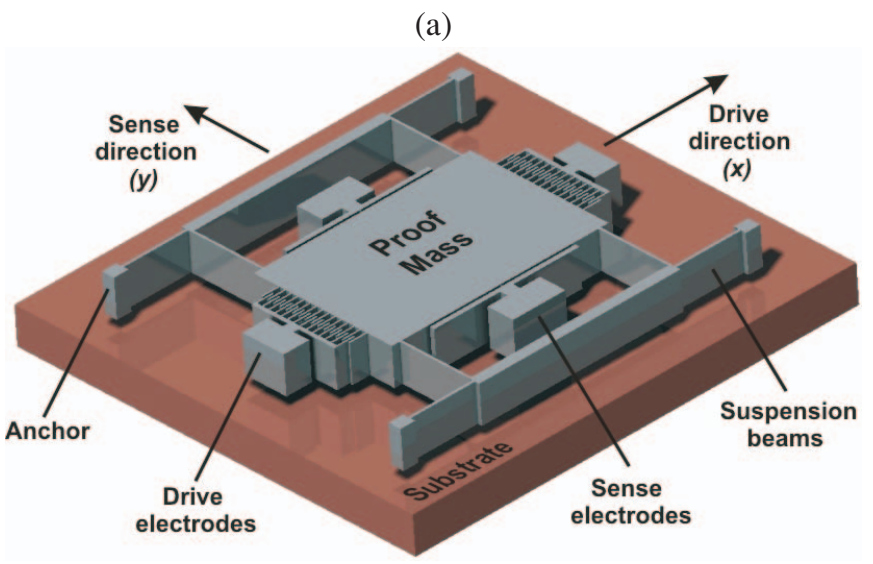

(b)

Fig. 3. (a) Theoretical Response of a classical Type II gyroscope; (b) A Design of Type II gyroscope 


\section{B. Design}

The drive and sense mode matching requirement in vibratory gyroscopes renders the system response very sensitive to variations in system parameters due to fabrication imperfections and fluctuations in operating conditions, which shift the drive or sense resonant frequencies. Fabrication tolerance often results in natural frequencies that are mismatched by a few percent.

For the devices packaged in vacuum to enhance the sensitivity, the bandwidth of the resonance peaks is extremely narrow; leading to much tighter mode-matching requirements. Extensive research has been focused on design of symmetric drive and sense-mode suspensions for mode-matching and minimizing temperature dependence. However, especially for lightly-damped devices, the mode-matching requirement is well beyond fabrication tolerances; and none of the symmetric designs can provide the required degree of mode-matching without feedback control.

Two major design concepts were explored by our group in order to achieve a dynamical system with a wide-bandwidth frequency response: expanding the system design space by (1) increasing the degrees-of-freedom (DOF) of the drive and sense mode oscillatory systems and (2) utilizing multiple drive-mode oscillators with incrementally spaced resonant frequencies [4], [2], [7].

1) Non-Resonant Vibratory Gyroscopes: One approach to increase bandwidth of a vibratory gyroscope is to design non-resonant multi-degree of freedom resonator, for example utilizing a 3-DOF dynamic system, as opposed to the 2-DOF system conventionally used in Type II MEMS gyroscopes. The 3-DOF design concept aims to utilize resonance in either the drive mode or the sense mode to improve sensitivity while maintaining the robust operational characteristics. This is achieved by forming, for example, structurally decoupled 2-DOF and 1-DOF oscillators in the drive and sense-modes, Figure 4. The 2-DOF oscillator frequency response has two resonance peaks, and a flat region between the peaks, that defines the operation frequency region, Figure 5. The 1DOF oscillator resonant frequency is designed to overlap with the drive-mode flat operating region. Thus, the device is operated at the resonant frequency of the 1-DOF oscillator for improved sensitivity, while 2-DOF oscillator amplitude is inherently constant within the same frequency band. We' have also considered an approach of defining a wide-bandwidth gyroscope by 3-DOF gyro structure with 1-DOF drive and 2-DOF sense modes. The results can be found in [4]

The detection and control electronics for Type II MVGs is generally simpler than for Type I MVGs, as the drive and response detection are fundamentally one dimensional. The sense and drive electronics for Type II devices are extensively discussed in the literature and is not covered here.

2) Distributed Mass Vibratory Gyroscopes: The design concept is based on forming multiple drive-mode oscillators, distributed symmetrically around the center of a supporting frame [6]. The distributed drive-mode oscillators are driven in-phase towards the center of symmetry, and are structurally

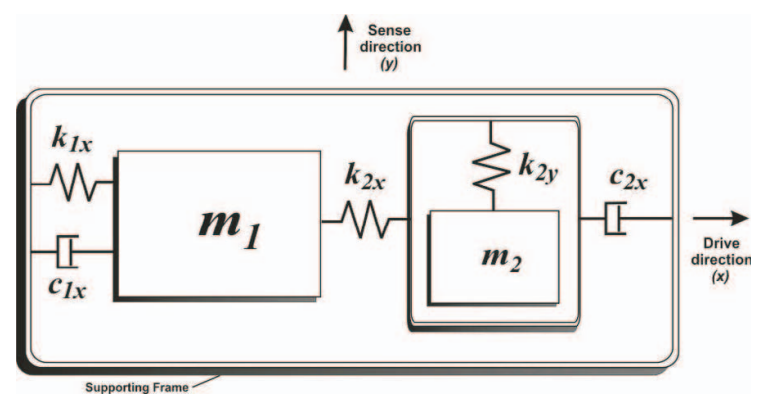

Fig. 4. Lumped mass-spring-damper model of the overall 3-DOF gyroscope dynamical system with 2-DOF drive-mode

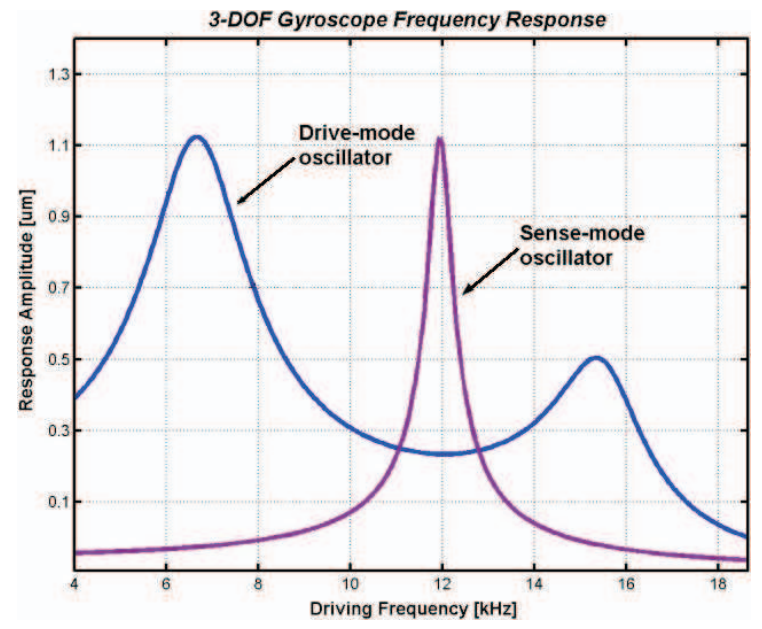

Fig. 5. The frequency responses of the 2-DOF drive and 1-DOF sensemode oscillators. The drive-direction oscillation amplitude is insensitive to parameter variations and damping fluctuations in the flat operating region, eliminating mode-matching requirement

constrained in the tangential direction with respect to the supporting frame. Each oscillator is driven at the same drive frequency. In the presence of an angular rotation rate about the z-axis, a sinusoidal Coriolis force at the drive frequency is induced on each proof mass in the direction orthogonal to each drive-mode oscillation directions (Figure 6. Thus, each of the induced Coriolis force vectors lie in the tangential direction, combining to generate a resultant torque on the supporting frame. The net Coriolis torque excites the supporting frame into torsional oscillations about the z-axis, which are detected by sense capacitors for angular rate measurement.

In the presented design concept, a wide-bandwidth operation region is achieved in the drive-mode frequency response, by designing or actively tuning the resonance frequency of each drive-mode oscillator to be incrementally spaced. Since the tangential Coriolis forces induced on each proof mass jointly generate a resultant torque on the supporting frame, a "levelled" total Coriolis torque is achieved over a wide range of driving frequencies. The device is nominally operated in this levelled region of the Coriolis torque frequency response, so that fluctuations in system parameters that shift oscillator resonance frequencies will not result in a significant change in the total Coriolis torque. If the sense-mode resonance fre- 


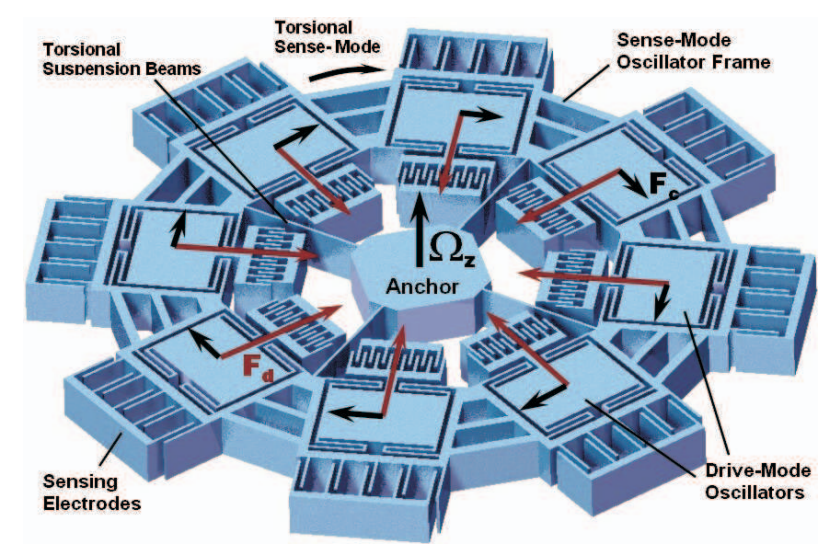

Fig. 6. Conceptual illustration of the Distributed-Mass Gyroscope with 8 symmetric drive-mode oscillators

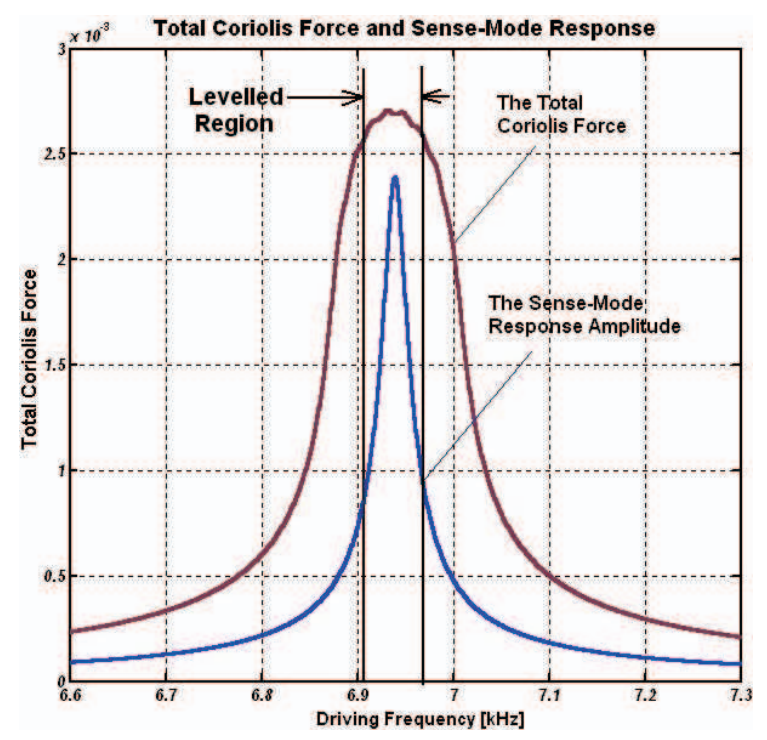

Fig. 7. The frequency spectrum of the total Coriolis torque generated by the distributed drive-mode oscillators

quency is designed to be accommodated in the same frequency band (Figure 7), the requirement on the degree of modematching is relaxed, and robustness against structural and thermal parameter fluctuations is achieved.

This design approach provides wider drive-mode bandwidth in vibratory MEMS gyroscopes. The approach was theoretically illustrated, and experimentally verified in [6].

\section{Results}

In this section the Non-Resonant Type II MVG is illustrated. The synchronous demodulation technique was utilized to extract the angular rate response of the 3-DOF system at different temperatures. The drive signal applied on the combdrive actuators was $25 \mathrm{~V}$ DC bias and $3 \mathrm{~V} \mathrm{AC}$. The drive-mode amplitude was continuously monitored during the operation of the device using a microscope attached to the rate-table platform. The temperature of the device also continuously monitored using a solid-state temperature sensor attached to the sample.

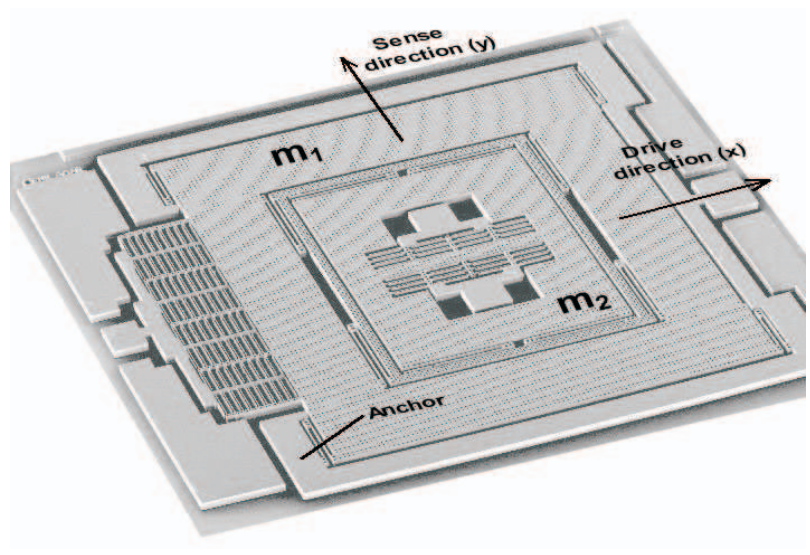

(a)

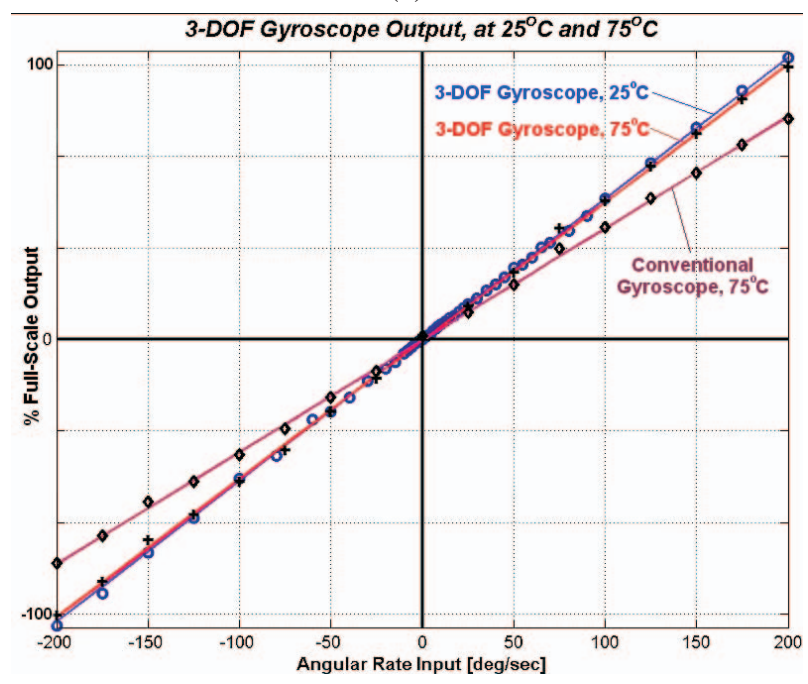

(b)

Fig. 8. (a) SEM image of non-resonant Type II gyroscope; (b) The angularrate response of the 3-DOF gyroscope with 2-DOF sense-mode, at $25^{\circ} \mathrm{C}$ and $75^{\circ} \mathrm{C}$.

A lock-in amplifier was utilized to provide the driving AC signal applied on the comb-drives, and to synchronously demodulate the Coriolis signal at the drive frequency in the internal reference mode. The output of the lock-in amplifier is low-pass filtered to provide a stable output reading proportional to the applied input angular rate.

When the temperature of the gyroscope was increased from $25^{\circ} \mathrm{C}$ to $75^{\circ} \mathrm{C}$ while keeping the excitation frequency constant at $752 \mathrm{~Hz}$, the sensitivity of the gyroscope was observed to drop from $0.0308 \mathrm{mV} / \% / \mathrm{s}$ to $0.0234 \mathrm{mV} / \% / \mathrm{s}$ (Figure $8(\mathrm{~b}$ )). This translates into $24.1 \%$ drop in the response gain. When the change in the drive-mode amplitude from $25^{\circ} \mathrm{C}$ to $75^{\circ} \mathrm{C}$ is investigated, it is seen that is changes from $5.8 \mu \mathrm{m}$ to $4.3 \mu \mathrm{m}$; yielding a $25.9 \%$ change. Thus, it is demonstrated that the change in the gyroscope sensitivity is almost exactly equal to the drive-mode amplitude change (with 1.8\% discrepancy); verifying the insensitivity of the sense-mode response to temperature variations.

In order to compare the improved robustness of the proposed 
wide-bandwidth approach, a micromachined gyroscope with a conventional 2-DOF dynamical system was characterized under the same temperature variations and using the same signal conditioning electronics.

When the temperature of the tested conventional gyroscope was increased from $25^{\circ} \mathrm{C}$ to $75^{\circ} \mathrm{C}$ while restoring the drivemode amplitude to $12 \mu \mathrm{m}$, the sensitivity was observed to drop from $0.91 \mathrm{mV} /{ }^{\circ} / \mathrm{s}$ to $0.73 \mathrm{mV} /{ }^{\circ} / \mathrm{s}$. Thus, a $50^{\circ} \mathrm{C}$ temperature increase was observed to result in $19.8 \%$ sensitivity change in the conventional gyroscope, which is over 12.2 times larger than the wide-bandwidth gyroscope approach.

The presented experimental results illustrate that the idea of the wide-band distributed mass Type II gyroscopes is feasible. The unique characteristics of the design idea may lead to a class of MEMS gyroscopes with the order of magnitude increase in the bandwidth and robustness without sacrifice in sensitivity.

\section{CONCLUSIONS}

We introduced and demonstrated Type I and Type II Micromachined Vibratory Gyroscopes.

For the first time, a Type I MVG has been realized and demonstrated. The devise was verified to be capable of measuring absolute angles of rotation directly, [3]. The results are preliminary, and future work will focus on understanding the factors effecting performance of Type I gyros.

We summarized two classes of wide-bandwidth Type II Micromachined Vibratory Gyroscopes. This topic was extensively studied in [4]. The demonstrated 3-DOF prototypes exhibited a noise-floor of $0.640 / s / v H z$ in atmospheric pressure, with the designed off-chip detection electronics based on synchronous demodulation. Rate table experiments at $25^{\circ} \mathrm{C}$ and $75^{\circ} \mathrm{C}$ showed only $1.62 \%$ change in the scale factor without active control, while conventional devices resulted in $19.8 \%$ scale factor change, verifying the improved robustness of the nonresonant Type II MVG design concept.

The micromachined gyroscopes of both types could potentially yield reliable, robust and high performance angularrate measurements leading to a wide range of applications including dynamic vehicle control, automotive safety systems, navigation/guidance systems, and interactive consumer electronics.

\section{ACKNOWLEDGMENT}

This work was partially supported by the National Science Foundation Grants CMS-0409923 and CMS-0449442, program director Dr. Shih-Chi Liu. This work was also cosponsored by BEI Technologies Inc. and the University of California Discovery Program. Chris Painter was partially supporting by the US Department of Defense through a 2001 National Defense Science and Engineering Graduate Fellowship.

\section{REFERENCES}

[1] A. M. Shkel and R.T. Howe "Surface Micromachined Rate Integrating Gyroscopes" US Patent No. 6,481,285
[2] C. Acar and A. M. Shkel "Non-Resonant Four Degrees-of-Freedom Micromachined Gyroscope" U.S. Patent No. 6,845,669

[3] C. Painter and A. M. Shkel "Multi Stage Control Architecture for Error Supression in Micromachined Gyroscopes" U.S. Patent No. 6,934,660

[4] C. Acar "Robust Micromachined Vibratory Gyroscopes" Ph.D. thesis, Dept. of MAE, University of California - Irvine 2004

[5] C. Painter "Micromachined Vibratory Gyroscopes with Imperfections" Ph.D. thesis, Dept. of MAE, University of California - Irvine 2005

[6] C. Acar and A. M. Shkel "An Approach for Increasing Drive-Mode Bandwidth of MEMS Vibratory Gyroscopes" Journal of Microelectromechanical Systems Vol. 14, No. 3, pp. 520-528, 2005

[7] C. Acar and A. M. Shkel "Non-Resonant Micromachined Gyroscopes with Mode-Decoupling" IEEE Sensors Journal vol. 3, No. 4, August 2003, pp. 497-506

[8] C. Painter and A. M. Shkel "Active Structural Error Supression in MEMS Vibratory Rate Integrating Gyroscopes" IEEE Sensors Journal Vol. 3, No.5, pp. 595-606, 2003

[9] N. Yazdi, F. Ayazi,and K. Najafi "Micromachined Inertial Sensors" Proceedings of the IEEE, volume 86, Number 8 pp. 1641-1659, August, 1998

[10] J. Soderkvist "Micromachined gyroscopes" Sensors and Actuators A volume 43, pp.65-71, 1994 